\title{
Polarimetry of the dwarf planet (136199) Eris ${ }^{\star}$
}

\author{
I. Belskaya ${ }^{1}$, S. Bagnulo ${ }^{2}$, K. Muinonen ${ }^{3}$, M. A. Barucci ${ }^{4}$, G. P. Tozzi ${ }^{5}$, S. Fornasier ${ }^{4,6}$, and L. Kolokolova ${ }^{7}$ \\ 1 Astronomical Observatory of Kharkiv National University, 35 Sumska str., 61022 Kharkiv, Ukraine \\ e-mail: irina@astron.kharkov.ua \\ 2 Armagh Observatory, College Hill, Armagh BT61 9DG, Northern Ireland, UK \\ e-mail: sba@arm.ac.uk \\ 3 Observatory, PO Box 14, 00014 University of Helsinki, Finland \\ e-mail: muinonen@cc.helsinki.fi \\ ${ }^{4}$ LESIA, Observatoire de Paris, 5 place Jules Janssen, 92195 Meudon Cedex, France \\ e-mail: [antonella.barucci; sonia. fornasier]@obspm. fr \\ 5 INAF - Oss. Astrofisico di Arcetri, Largo E. Fermi 5, 50125 Firenze, Italy \\ e-mail: tozzi@arcetri.astro.it \\ 6 University of Paris 7 "Denis Diderot”, 10 rue Alice Domon et Leonie Duquet, 75013 Paris, France \\ 7 University of Maryland, College Park, MD, USA \\ e-mail: ludmilla@astro.umd.edu
}

Received 7 July 2007 / Accepted 23 November 2007

\section{ABSTRACT}

\begin{abstract}
Context. We study the surface properties of the transneptunian population of Solar-system bodies. Aims. We investigate the surface characteristics of the large dwarf planet (136199) Eris.

Methods. With the FORS1 instrument of the ESO Very Large Telescope, we have obtained Bessell broadband $R$ linear polarimetry and broadband $V$ and $I$ photometry of Eris. We have modelled the observations in terms of the coherent-backscattering mechanism to constrain the surface properties of the object.

Results. Polarimetric observations of Eris show a small negative linear polarization without opposition surge in the phase angle range of $0.15^{\circ}-0.5^{\circ}$. The photometric data allow us to assume a brightness opposition peak at phase angles below $0.2^{\circ}-0.3^{\circ}$. The data obtained suggest a possible similarity to the polarimetric and photometric phase curves of Pluto. The measured absolute magnitude and broadband colors of Eris are $H_{V}=-1.15 \mathrm{mag}, V-R=0.41 \mathrm{mag}$, and $V-I=0.75 \mathrm{mag}$.

Conclusions. The observational data with theoretical modelling are in agreement with the surface of Eris being covered by large inhomogeneous particles.
\end{abstract}

Key words. Kuiper Belt - polarization - scattering

\section{Introduction}

The discovery of the Pluto-sized transneptunian object $2003 \mathrm{UB}_{313}$ (Brown et al. 2005), later named (136199) Eris, was succeeded by an intense physical characterization phase, and no less than a discussion on the definition of a planet. After the resolution of IAU (24 August 2006) Eris is classified, together with Pluto, as a member of the new category of "the dwarf planets". Eris was discovered near its aphelion at 97 AU from the Sun and has an orbit with high eccentricity (0.44) and inclination $\left(44^{\circ}\right)$. According to its dynamical characteristics, it belongs to the so-called "detached objects" with pericenters decoupled from Neptune (Gladman et al. 2007).

The first size estimation of Eris was carried out by Bertoldi et al. (2006) based on thermal emission, resulting in a diameter of $3000 \pm 400 \mathrm{~km}$ and a geometric albedo of $0.60 \pm 0.15$. Later on, Brown et al. (2006), using the Hubble Space Telescope, measured directly the angular size of Eris corresponding to the diameter of $2400 \pm 100 \mathrm{~km}$, about $5 \%$ larger than that of Pluto, and derived the albedo of $0.87 \pm 0.05$ in the $V$ band. The

\footnotetext{
* Based on observations made with ESO Telescopes at the Paranal Observatory under programme ID 178.C-0036 (PI: A. Barucci).
}

discrepancy in the albedo is rather large but, undoubtedly, Eris is a high-albedo object like Pluto (see Buratti et al. 2003). According to Brown \& Schaller (2007), the mass of Eris exceeds the mass of Pluto by a factor of 1.27.

The spectral properties of Eris are also found to be similar to those of Pluto. The near-infrared spectrum is dominated by methane ice absorptions (Brown et al. 2005). However, the central wavelengths of the $\mathrm{CH}_{4}$ bands do not show the shift in wavelength seen in the spectra of Pluto and Triton, which is attributed to the fact that most (Triton) or some (Pluto) of the $\mathrm{CH}_{4}$ is dissolved in solid $\mathrm{N}_{2}$. The quality of Eris' spectrum does not allow the detection of $\mathrm{N}_{2}$. Licandro et al. (2006) reported a visible spectrum of Eris in which the $\mathrm{CH}_{4}$ ice bands show a small shift in the band at around $0.89 \mu \mathrm{m}$ and they suggested that the shift could indicate the presence of methane diluted in $\mathrm{N}_{2}$. Dumas et al. (2007), using the new SINFONI instrument installed at the ESO Very Large Telescope (VLT), obtained a high-quality nearinfrared spectrum and, on the basis of modelling, they obtained the best fit of the spectrum including $\mathrm{N}_{2}$ on the surface of Eris. Due to the highly eccentric orbit, they expect a thin atmosphere close to perihelion, which freezes out onto the surface when the object is located at a larger heliocentric distance. They modelled 
Table 1. Polarimetry of (136199) Eris in the Bessell $R$ band. $P_{Q}$ and $P_{U}$ are the Stokes parameters transformed such that $P_{Q}$ represents the flux perpendicular to the plane Sun-Object-Earth (the scattering plane) minus the flux parallel to that plane, divided by the sum of the two fluxes, and $\Theta$ is the position angle measured counterclockwise from the perpendicular to the scattering plane.

\begin{tabular}{cccccrr}
\hline \hline $\begin{array}{c}\text { Date } \\
\text { (yyyy mm dd) }\end{array}$ & $\begin{array}{c}\text { Time (UT) } \\
\text { (hh:mm) }\end{array}$ & Sky & $\begin{array}{c}\text { Phase angle } \\
\text { (DEG) }\end{array}$ & $\begin{array}{c}P_{Q} \\
(\%)\end{array}$ & \multicolumn{1}{c}{$\begin{array}{c}P_{U} \\
(\%)\end{array}$} & \multicolumn{1}{c}{\begin{tabular}{c} 
(DEG) \\
\hline 20061019
\end{tabular}} \\
$03: 27$ & PHO & 0.15 & $-0.09 \pm 0.07$ & $0.00 \pm 0.07$ & $91 \pm 23$ \\
20061112 & $02: 21$ & CLR & 0.30 & $-0.06 \pm 0.07$ & $-0.12 \pm 0.07$ & $122 \pm 19$ \\
20061118 & $02: 25$ & THN & 0.35 & $-0.32 \pm 0.12$ & $0.00 \pm 0.10$ & $90 \pm 9$ \\
20061214 & $01: 53$ & PHO & 0.51 & $-0.12 \pm 0.07$ & $-0.05 \pm 0.07$ & $100 \pm 21$ \\
\hline
\end{tabular}

the surface using two types of terrains of distinct composition: about $50 \%$ covered with pure methane ice, while the other part is made of a mixture of methane, nitrogen, and water and tholin ices. The best model fits are obtained when the icy grains are rather large, from sub-mm to a few tens of $\mathrm{mm}$ in size (Dumas et al. 2007).

In the present paper, we report the first polarimetric observations of Eris made as part of the Large-Program observations at ESO VLT, intended to set constraints on its surface properties.

\section{Observations and data reduction}

Observations of Eris have been obtained at the ESO VLT with the FORS1 instrument in service mode during an observing period from October to December 2006.

FORS1 is a multi-mode instrument for imaging and spectroscopy equipped with polarimetric optics. For the present study, FORS1 has been used to measure the broadband linear polarization of Eris at four different epochs, in the Bessell $R$ filter. Additional photometric observations were made in the $V$ and $I$ filters.

Taking advantage of the flexibility offered by the VLT service observing mode, we distributed the observations along three months to obtain data points in the maximum possible range of phase angles for the ground-based observations, setting precise time intervals for the execution of the observations. To reduce the impact of sky background on the data quality, we generally tried to avoid observations with the target close to the Moon, and with a large fraction of lunar illumination.

Polarimetric observations were performed with the retarder waveplate at all positions between $0^{\circ}$ and $157.5^{\circ}$ (at $22.5^{\circ}$ steps), setting the exposure time of each frame to $180 \mathrm{~s}$. The frames were then processed and combined as explained in Bagnulo et al. (2006). In addition to the data reduction procedure described in Bagnulo et al. (2006), the observed position angle was rotated for Eris by $-1.23^{\circ}$ to compensate for a small chromaticity effect of the instrument retarder waveplate. Further details can be found in the FORS1 user manual and in Fossati et al. (2007).

The errors were calculated taking into account both photon noise and background subtraction. We found that, for apertures of about 7-8 pixels, the error due to the photon noise was similar to the error introduced by the background subtraction (for smaller apertures, the former is the dominating source of error, and vice versa). From the observations obtained on 2006-10-19, 2006-11-12, and 2006-12-14, we measured a polarization that was generally independent of the aperture. For these observations, we adopted the polarization measured for apertures of 6 to 8 pixels (note that, for such small aperture values, the fluxes measured in the ordinary and extra-ordinary beams still increase with the aperture). In contrast, from the observations taken on 2006-11-18 we found that the polarization depends strongly on
Table 2. Photometry of (136199) Eris. The errors on $R$ magnitudes are estimated a priori (see text).

\begin{tabular}{ccccc}
\hline \hline $\begin{array}{c}\text { Date } \\
\text { (yyy mm dd) }\end{array}$ & $\begin{array}{c}\text { Time (UT) } \\
\text { (hh:mm) }\end{array}$ & $\begin{array}{c}\text { Phase angle Band } \\
\text { (DEG) }\end{array}$ & & Magnitude \\
\hline 20061019 & $03: 27$ & 0.15 & $R$ & $18.34 \pm 0.05$ \\
20061020 & $05: 42$ & 0.16 & $V$ & $18.74 \pm 0.02$ \\
20061020 & $05: 45$ & 0.16 & $I$ & $18.00 \pm 0.02$ \\
20061020 & $05: 49$ & 0.16 & $V$ & $18.75 \pm 0.02$ \\
20061020 & $05: 52$ & 0.16 & $I$ & $17.99 \pm 0.02$ \\
20061112 & $02: 21$ & 0.30 & $R$ & $18.37 \pm 0.05$ \\
20061214 & $01: 53$ & 0.51 & $R$ & $18.40 \pm 0.05$ \\
\hline
\end{tabular}

the aperture, varying from about $0.2 \%$ for a 5 pixel aperture, up to about $0.6 \%$ for an aperture of 20 pixels. We found that by discarding the frame obtained at $22.5^{\circ}$, the polarization is more independent of the aperture, varying from about $0.1 \%$ for a 5 pixel aperture, to about $0.4 \%$ for a 20 pixel aperture. However, we could not find anything obviously wrong with the raw data obtained with the retarder waveplate at $22.5^{\circ}$. For the point obtained on 2006-11-18, we finally adopted a value of about $0.3 \%$, which is consistent with the results obtained by discarding the frame obtained at $22.5^{\circ}$.

The results of polarimetric observations are given in Table 1, which contains the epoch of the observations (date and UT time), the night-time sky conditions $(\mathrm{THN}=$ thin cirrus, CLR $=$ clear, $\mathrm{PHO}=$ photometric), the phase angle, the measured Stokes parameters $P_{Q}$ and $P_{U}$, and the position angle of the linear polarization $\Theta$, transformed relative to the scattering plane as explained in Bagnulo et al. (2006) and Landi Degl'Innocenti et al. (2007).

The linear polarization of the light scattered by the surface of Eris in the phase-angle range of $0.15-0.51^{\circ}$ is found to be small, never exceeding a $3 \sigma$ detection.

Acquisition images were used to obtain photometry, although not all observing nights were clear. In particular, bad sky transparency during the night of 2006-11-18 did not allow us to obtain a proper estimate of the magnitude. We associated an error of 0.05 mag to the photometry, which is consistent with the zero points of the calibration plan, with the errors of photon noise and background being negligible in comparison with those of the zero points. Additional photometric observations in the $V$ and $I$ bands were obtained during the photometric night of 2006-10-20 at the phase angle of $0.16^{\circ}$, close to the minimum phase angle reachable for Eris in this apparition. The integration times in the $V$ and $I$ bands were $100 \mathrm{~s}$ and $200 \mathrm{~s}$, respectively, in order to obtain high-signal-to-noise-ratio images. The images were taken when the object was at the airmass of 1.13. The data were reduced in a standard manner described in detail by Peixinho et al. (2004). Results of photometric measurements are given in Table 2. They allowed for the estimation of the 


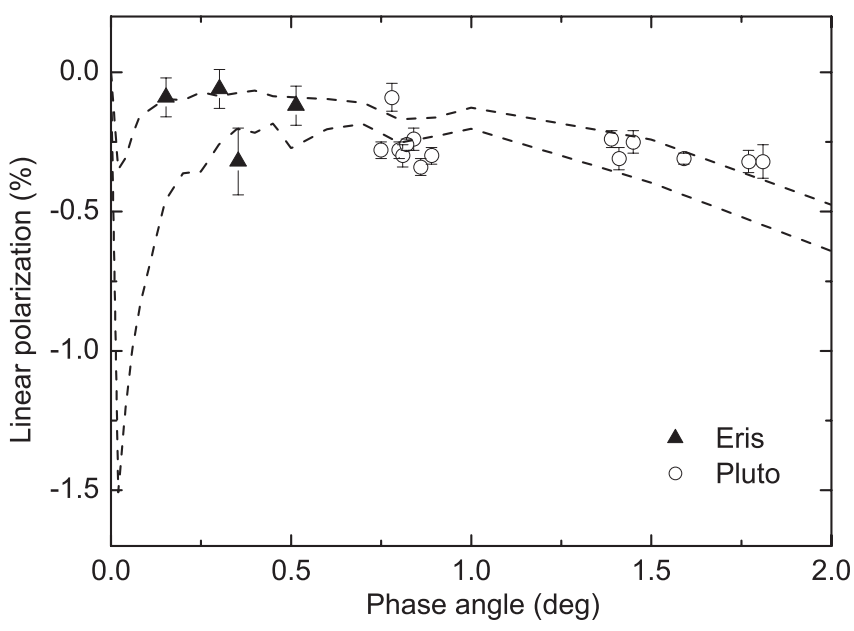

Fig. 1. Linear polarization observations vs. phase angle for Eris and Pluto (for references, see text). The dashed curves are the results of our modelling and show the envelope of the polarization curves for the given range of geometric albedos (see Sect. 3). The Eris $0.35^{\circ}$ point was not included in the derivation of the envelope.

absolute magnitude and broadband colors of Eris: $H_{V}=$ $-1.15 \mathrm{mag}, V-R=0.41 \mathrm{mag}$, and $V-I=0.75 \mathrm{mag}$.

\section{Results and discussion}

Polarimetry is a powerful tool to investigate the physical properties of solid surfaces of Solar-system bodies. At small phase angles, these objects typically exhibit a phenomenon of negative polarization. It is a peculiar case of partially linear polarized scattered light, where the electric field vector component parallel to the scattering plane predominates over the perpendicular component. Negative linear polarization was first discovered by Lyot (1929) for the Moon and later found to be a ubiquitous phenomenon for planetary surfaces at small phase angles. The linear polarization varies with the phase angle, characterizing the properties of the surface, such as particle size, heterogeneity, complex refractive index, porosity, and surface roughness. The first polarimetric observations for a Trans Neptunian Object (except Pluto) carried out by Boehnhardt et al. (2004) for (28978) Ixion revealed a pronounced negative polarization changing noticeably as a function of the phase angle, in spite of the small range of phase angles covered $\left(0.2^{\circ}-1.3^{\circ}\right)$. Since then, three other objects have been observed: (2060) Chiron and (50000) Quaoar (Bagnulo et al. 2006) and 29981 (1999 TD 10 ) (Rousselot et al. 2005). The polarimetric data available for TNOs show noticeable negative polarization with two different trends at small phase angles: subtle changes in the negative polarization are observed for the largest objects Pluto and Quaoar, whereas sudden enhancements are observed for the others. Our observations of Eris do not show noticeable changes of linear polarization in the phase angle range covered. Figure 1 shows the polarimetric observations of Eris in the $R$ band, and Pluto in the visible light, versus phase angle. Note that the observations of Pluto refer to the Pluto-Charon system and show notable similarity in the degree of polarization measured in different oppositions by different authors (Kelsey \& Fix 1973; Breger \& Cochran 1982; Avramchuk et al. 1992). Unfortunately, the observations of Eris and Pluto cover different ranges in the phase angle. However, the objects can be anticipated to be similar in their surface characteristics.

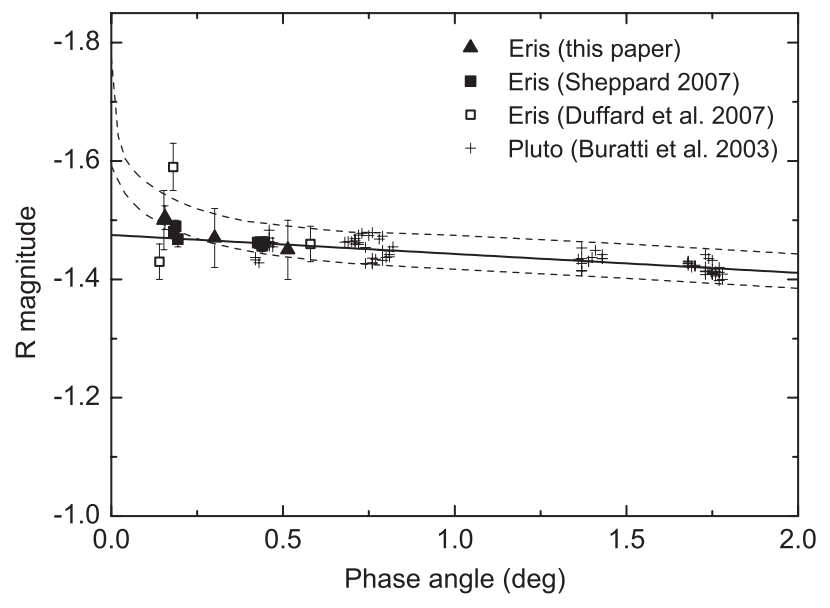

Fig. 2. Reduced $R$ magnitude vs. phase angle for Eris. Data of Pluto in the $R$ band is plotted according to Buratti et al. (2003) with an arbitrary shift to the Eris data. The solid line shows a linear fit to all data. The dashed lines show the envelope of the photometric model curves derived simultaneously with the polarimetric envelope.

The small linear polarization near opposition for a bright object like Eris is somewhat unexpected. According to the mechanism of coherent backscattering, which is considered as the most probable cause of negative polarization at small phase angles (for reviews, see Muinonen et al. 2002; Belskaya et al. 2007), a sharp asymmetric surge of negative polarization is expected for high-albedo objects. Rosenbush et al. (1997, 2005) report detections of such surges for bright satellites and asteroids. In all cases, the surges are accompanied by narrow brightness opposition peaks in agreement with coherent-backscattering modelling (for a review, see Mishchenko et al. 2006).

Figure 2 shows the magnitude phase dependence in the $R$ band for Eris in comparison with observations of Pluto in the same band plotted according to Buratti et al. (2003). The magnitude phase dependence for Eris was composed of our observations (see Table 2), those from Sheppard (2007) in 2005 averaged for each observing night, and those from Duffard et al. (2007) in 2005-2007 averaged for each observing run. All observations agree within their uncertainties. The scatter of the data is probably connected with rotational brightness variations of Eris, which are impossible to take into account since the rotation period remains unknown. According to Duffard et al. (2007), a short-term variability is very low but a very long rotation period cannot be ruled out. The data available show that a long-term variability of Eris (if it exists) should be less than $0.1 \mathrm{mag}$, contrary to Pluto with a lightcurve amplitude of $0.33 \mathrm{mag}$ and a rotation period of 6.387 days (Buie et al. 1997). The data for Pluto in Fig. 2 were shifted with respect to the data for Eris in order to make the phase curves coincide in the overlapping phase-angle range. As in the case of polarimetric phase dependence (Fig. 1), both objects show similar trends with phase angle. The linear phase coefficient derived for Pluto in the phase angle range of $0.4^{\circ}-1.8^{\circ}$ is $0.032 \pm 0.001 \mathrm{mag} / \mathrm{deg}$ (Buratti et al. 2003). A linear fit to the Eris data in the phase-angle range of $0.2^{\circ}-0.6^{\circ}$ gave a phase coefficient of $0.08 \pm 0.03 \mathrm{mag} / \mathrm{deg}$. Rabinowitz et al. (2007) reported a similar small phase coefficient of $0.10 \pm 0.02 \mathrm{mag} / \mathrm{deg}$ in the $V$ band. However, all these estimations are rather uncertain due to large scatter of data and the small range of the covered phase angles. In fact, data of Pluto and Eris can be fitted by a single linear dependence with Pluto's 
phase coefficient (see solid line in Fig. 2). A small increase in brightness toward opposition found for Eris is unusual for bright surfaces typically showing a pronounced opposition surge with a phase slope of $0.2-0.3 \mathrm{mag} / \mathrm{deg}$ in the same phase range of $0.2-0.6^{\circ}$ (e.g. Verbiscer et al. 2005). An opposition surge can exist at smaller phase angles not covered by the observations.

According to the data available, the photopolarimetric properties of Eris and Pluto are expected to be quite similar. When interpreting the data, we need to take into account the existence of a thin atmosphere of Pluto and possible thin atmosphere of Eris. As compared to the satellites of the major planets having atmospheres, the absence of negative polarization at small phase angles has been reported for Titan only (Veverka 1973), while for Io and Europa, the negative polarization peak was found with an amplitude of about $0.4 \%$ at phase angles of $0.2-0.7^{\circ}$ (Rosenbush $\&$ Kiselev 2005). The absence of such a peak for Eris puts constraints on its surface properties.

Another point that needs to be mentioned is that both objects have satellites. While the satellite of Eris is small (Brown et al. 2006) and should not affect the observations made, all data discussed above for Pluto refer to the Pluto-Charon system. Buie et al. (1997) made an attempt to separate the photometric properties of these objects and obtained a smaller phase coefficient for Pluto $(0.029 \mathrm{mag} / \mathrm{deg})$ as compared to the Pluto-Charon system (0.032 mag/deg). The contribution of Charon to the linear polarization observed for the Pluto-Charon system remains unknown.

Below, we present results of modelling of the photopolarimetric properties of Eris and the Pluto-Charon system, assuming similar behaviors for their photometric and polarimetric phase curves.

We explain the polarimetric phase curves of Eris and Pluto through numerical simulations of coherent backscattering by Rayleigh scatterers in spherical media (Muinonen 2004), assuming a model composed of two kinds of scattering media (e.g., Boehnhardt et al. 2004; Bagnulo et al. 2006). There are five parameters in the model: two single-scattering albedos, two mean free paths, and the weight factor $w_{\mathrm{d}}$ for the darker component (the weight factor of the brighter component being $w_{\mathrm{b}}=1-w_{\mathrm{d}}$ ).

We make use of existing computations for 630 spherical random media: the database entails 21 single-scattering albedos $\tilde{\omega}=0.05,0.10, \ldots, 0.90,0.95,0.97,0.99$, and 30 dimensionless mean free paths $k \ell=2 \pi \ell / \lambda=10,20,30, \ldots, 100,120,140$, $\ldots, 200,250,300, \ldots, 400,500,600, \ldots, 1000,2000,3000$, $\ldots, 5000,10000$ ( $k$ and $\lambda$ are the wave number and wavelength). The spherical media have, in essence, infinite diametrical optical thicknesses, mimicking macroscopic objects.

Figure 1 shows the envelope of the extreme values of polarization curves when fixing the model geometric albedo at $p_{R}=0.600,0.601,0.602, \ldots, 0.900$, and, for each albedo, updating the polarization envelope at each phase angle if the rms is less than 0.12 (roughly twice the observational error). Note that the boundaries of the envelope do not correspond to any single model polarization curve, but rather outline the regime of plausible curves. The Eris observation at the phase angle of $0.35^{\circ}$ was omitted as an outlier. Some of the polarimetric observations of Pluto are located on or outside the boundaries of the envelope computed. We consider this systematic feature to be mainly due to the limitations of the two-component Rayleigh-scatterer model utilized.

A photometric opposition-effect envelope was derived in a way resembling the derivation of the polarimetric envelope. The photometric envelope is defined, at a given phase angle, by the maximum and minimum ratios of disk-integrated brightness at that phase angle and at exact opposition. In order to obtain reasonable fits, the coherent-backscattering peaks were multiplied by an additional linear-exponential function with a peak width and amplitude of $0.2^{\circ}$ and $10 \%$, respectively (cf. Rousselot et al. 2005). This additional enhancement can be envisaged as arising from shadowing contributions. Alternatively, the need for the additional function could be removed through more sophisticated coherent-backscattering modelling. In Fig. 2, the photometric envelopes are depicted after introducing a small separation into the envelope curves for better illustration.

Based on the envelopes above and the opposition surge suggested in linear polarization and brightness phase curves at extremely small phase angles, we can conclude that the mean free paths in the regoliths of Eris and Pluto are probably long, potentially so long that the related opposition phenomena cannot be observed any more. For realistic high-albedo regolith structures, long mean free paths suggest that the scattering centers are located far from each other. One of the explanations of this finding may be that the regolith particles are quite transparent large particles with internal or surface inhomogeneities responsible for the coherent-backscattering phenomena. Such an explanation is in agreement with the spectroscopic findings reviewed above.

\section{Conclusions}

The present polarimetric observations of Eris constitute the first observations of a high-albedo transneptunian object at very small phase angles. A sharp surge of negative polarization expected for high-albedo objects within the mechanism of coherent backscattering was not found in the measured range of phase angles $\left(0.15-0.5^{\circ}\right)$. It was also shown that, if a brightness opposition peak exists for Eris, it must be constrained to phase angles below $0.2-0.3^{\circ}$. The data obtained for Eris suggest possible similarity with the polarimetric and photometric phase curves of Pluto. Their modelling puts constraints on the surface properties suggesting that the regoliths of Eris and Pluto probably consist of transparent inhomogeneous particles that are large compared to the wavelengths of visible light.

\section{References}

Avramchuk, V. V., Rakhimov, V. I., Chernova, G. P., \& Shavlovskii, N. M. 1992, Kinemat. Fiz. Nebesn. Tel, 8, N4, 37

Bagnulo, S., Boehnhardt, H., Muinonen, K., et al. 2006, A\&A, 450, 1239

Belskaya, I. N., Levasseur-Regourd, A.-C., Shkuratov, Yu, G., \& Muinonen, K. 2007, in The Solar System Beyound Neptune, ed. A. Barucci, et al. (Tucson, Arizona, USA: University of Arizona Pres), in press

Bertoldi, F., Altenhoff, W., Weiss, A., Menten, K. M., \& Thum, C. 2006, Nature, 439, 563

Boehnhardt, H., Bagnulo, S., Muinonen, K., et al. 2004, A\&A, 415, L21

Breger, M., \& Cochran, W. D. 1982, Icarus, 49, 120

Brown, M. E., \& Schaller, E. L. 2007, Science, 316, 1585

Brown, M. E., Trujillo, C. A., \& Rabinowitz, D. L. 2005, ApJ, 635, L97

Brown, M. E., Schaller, E. L., Roe, H. G., Rabinowitz, D. L., \& Trujillo, C. A. 2006, ApJ, 643, L61

Buie, M., Tholen, D., \& Wasserman, L. 1997, Icarus, 125, 233

Buratti, B. J., Hillier, J. K., Heinze, A., et al. 2003, Icarus, 162, 171

Carraro, G., Maris, M., Bertin, D., \& Parisi, M. G. 2006, A\&A, 460, L39

Duffard, R., Ortiz, J. L., Santos Sanz, P., et al. 2007, A\&A, submitted

Dumas, C., Merlin, F., Barucci, A., et al. 2007, A\&A,471, 331

Fossati, L., Bagnulo, S., Mason, E., \& Landi Degl'Innocenti, E. 2007, in The future of photometric, spectrophotometric, and polarimetric standardization, ed. C. Sterken, ASP Conf. Ser., 364, 503

Gladman, B., Marsden, B. G., \& VanLaerhoven, C. 2007, in The Solar System Beyound Neptune, ed. A. Barucci (Tucson, Arizona, USA: University of Arizona Press), in press

Kelsey, J. D., \& Fix, L. A. 1973, AJ, 184, 633 
Landi Degl'Innocenti, E., Bagnulo, S., \& Fossati, L. 2007, in The future of photometric, spectrophotometric, and polarimetric standardization, ed. C. Sterken, ASP Conf. Ser., 364, 495

Licandro, J., di Fabrizio, L., Pinilla-Alonso, N., de León, J., \& Oliva, E. 2006, A\&A, 457, 329

Lyot, B. 1929, Ann. Obs. Paris, 8, 1

Mishchenko, M. I.,Rosenbush, V. K., \& Kiselev, N. N. 2006, Applied optics, 45, 4459

Muinonen, K. 2004, Waves in Random Media, 14, 365

Muinonen, K., Piironen, J., Shkuratov, Yu. G., Ovcharenko, A., \& Clark, B. E. 2002, in Asteroids III, ed. W. Bottke, R. P. Binzel, A. Cellino, \& P. Paolicchi (Tucson, Arizona, USA: University of Arizona Press), 123

Peixinho, N., Boehnhardt, H., Belskaya I., et al. 2004, Icarus, 170, 153

Rabinowitz, D., Schaefer, B., \& Tourtellote, S. 2007, AJ, 133, 26
Rosenbush, V. K., \& Kiselev, N. N. 2005, Icarus, 179, 490

Rosenbush, V., Avramchuk, V., Rosenbush, A. E., \& Mishchenko, M. 1997, AJ, 487, 402

Rosenbush, V. K., Kiselev, N. N., Shevchenko, V. G., et al. 2005, Icarus, 178, 222

Rousselot, P., Levasseur-Regourd, A. C., Muinonen, K., \& Petit, J.-M. 2005 , Earth, Moon, Planets, 97, 353

Sheppard, S. S. 2007, AJ, 134, 787

Shkuratov, Yu., Ovcharenko, A., Zubko, E., et al. 2002, Icarus, 159, 396

Stansberry, J. A., Cruikshank, D. P., Grundy, W. G., et al. 2007, in The Solar System Beyound Neptune, ed. A. Barucci (Tucson, Arizona, USA: University of Arizona Press), in press

Verbiscer, A. J., French, R. C., \& Helfenstein, P. 2005, BAAS, 37, 702

Veverka, J. 1973, Icarus, 18, 657 